\title{
Using Dynamic Environments in Foreign Language Teaching
}

\author{
Yasin Aslan * \\ Sinop University, Sinop, Turkey
}

\begin{abstract}
With the advent of virtual environments, challenges and opportunities for innovative teaching and enhancement of student learning can be conducted more efficiently. What is important to this process is the use of strategies to foster transfer of knowledge generation dispositions from teacher to learner. The concept of leveraging strategic control of the knowledge from teachers to students in virtual learning environments functions as the basis for determining how to make students apply knowledge subsequent to the acquisition of knowledge. Learners of this century are born into digital world. They connect, share and learn by means of latest advances in technology. One of these innovations is social media which embrace web-based and mobile-based technologies to facilitate interactive communication between individuals, institutions, and communities. The Virtual Classroom enables an online learning environment to utilize a learning management system by which the teacher provides course content using a variety of resources and tools. Therefore it provides effective means of teaching students who are dispersed across different locations. Instructional strategies for fostering student engagement in foreign language classrooms are regarded as critical and a variety of theories focus on student learning, needs and adult concerns that provide a basis for transfer of knowledge from teacher to learner. In this study, a recent model which is composed of the dimensions of knowledge, teaching approach and knowledge authority is presented to design virtual learning environments in foreign language teaching. The study was carried out in Selcuk University, School of Foreign Languages in Konya. The results of the study show that dynamic virtual environments are quite significant in foreign language education.
\end{abstract}

Key Words: Virtual environments, Knowledge transfer, Foreign language learning

\section{Introduction}

Generally a teacher has been considered "an educational authority" and a "dispenser of knowledge". However, with the integration of ICT, knowledge resources have become accessible to the student in ways that are beyond teacher's control. In view of the fact that the students are usually regarded as "digital natives" or "screen generation," the teachers" traditional role has been reconsidered by educational researchers.

The most effective way to learn a language is to participate in a community in which the target language is used to communicate in a real context. In such an environment, the language learners are left with no place to hide. They are forced and encouraged to think, 
speak, and write in the target language. In other words, they become immersed in an inputrich, natural, and meaningful context in which the target language can be acquired spontaneously.

The evaluation of dynamic environments in education orginated fom the inspirations of Vygotsky's major concern for instruction and assessment. Vygotsky (1986) investigated the issue of instruction and assessment in the zone of proximal development in that the collaborative activity between two individuals in a purposeful manner would make a difference in the development and learning of the students. Luria (1961) introduced the idea of Dynamic Assessment to the western research community. The concept merged the instruction and assessment to reemerge then as a learning assessment procedure in that neither the instruction nor the assessment part of the DA could be exactly distinguished. Poehner and Lantolf (2005) stated that the unit of analysis in ZPD-sensitive activities is "the interpersonal functional system formed by people and cultural artifacts jointly to bring about development" (p. 238). Valinser and van der Veer (1993) believe that "all development involves in the construction of distance between the present and the past, and overcoming the distance from the present and the future" (p. 266).

The dynamic assessment aims at assessing the abilities of the learners through transforming them through dialogic activities between the learner and the mediator (Zhang, 2013). Indeed it is the interactiveness of the process sensitive to learners' responsiveness as well as metacognitive awareness that differentiates DA from other models of assessment and instruction (Haywood, 1992).

In foreign language education, Poehner and Lantolf (2005) also supported the implementation of the DA approach. Even though DA integrates the instruction and assessment, its objective is to realize the individual's "full picture" of cognitive ability in the field of second language learning.

Shortly after virtual environments have been popular, challenges and opportunities for innovative teaching and enhancement of student learning can be conducted more efficiently. What is important to this process is the use of strategies to foster transfer of knowledge generation dispositions from teacher to learner.

The concept of leveraging strategic control of the knowledge from teachers to students in virtual learning environments functions as the basis for determining how to make students apply knowledge subsequent to the acquisition of knowledge. Instructional strategies for fostering student engagement in foreign language classrooms are regarded as critical and a variety of theories focus on student learning, needs and adult concerns that provide a basis for transfer of knowledge from teacher to learner.

ELT teachers try to seek ways to make different tools available for students to be involved in their learning. CALL has played a significant role in the language learning context.

New tools such as mobile phones, podcasts and social networking have emerged in the field to have their effect on the learning mode. Online and Blended learning has proved to be a vital instrument for increasing learners' motivation and satisfaction. 


\section{Computer Assisted Language Learning (CALL)}

It is essential to make a profound analysis of Web as a language learning environment, not only due to the novelty of associated technologies (social networks, virtual worlds, Computer Mediated Communication, etc.), but also of the existence of versatile contents and usual lack of quality control for such contents, among other drawbacks (Felix, 2003).

CALL promotes personalized instruction and individualization in language learning, which, consequently, can increase learners' motivation because students direct much of the learning themselves. CALL allows language teachers to process and present authentic materials with flexibility.

\section{EFL Podcasting}

Podcasting as well as other newer web tools of wikis, twittering, and blogs have shown positive perceptions of utility, use and receptiveness of the technology application.

\section{Interaction and Integration with CALL}

Interaction is one of the key features of CALL which is provided in 3 ways: interpersonal interaction, computer-learner interaction, and intrapersonal interaction. Interpersonal interaction refers to interaction between learners and teachers, in which the latter negotiate the meaning of a linguistic form, co-construct its meaning, and are prompted to pay attention to the form. Computer-learner interaction refers to interaction between the learner and the computer program by clicking, typing, and recording after listening or observing multimedia material. Intrapersonal interaction concerns interaction that takes place within learners' mind, which stimulates their inner speech and engages their attention to linguistic forms and cognitive processing of input.

Integrating new CALL technology has transformed the medium of face-to-face to e-learning to c-learning (communicative learning, collaborative learning, constructivist learning, or community learning). There is a growing trend of digital culture in the current generation of learners.

\section{Blended learning}

Blended environments that provide multiple modalities for learning are preferred by increasing numbers of students. It has to be considered as a basic redesign of the teaching method with certain characteristics: a shift from lecture into existing teaching programs creates challenges for both teachers and learners. Students are conceived to show better computer skills than teachers- a fact that could both facilitate and challenge teachers' role in the classroom. Teachers could either choose to integrate their students' digital skills in the classroom activities or feel that their role as controllers of knowledge is jeopardized by new webogogical skills that they do not master.

\section{Mobile Assisted Language Learning (MALL)}

Mobile learning is pervasive as it gives an abundance of networked mobile and embedded devices, and ambient, in the sense, it is completely around us with learning 
enhanced to student-centered instruction in which students become active and interactive learners; increased interaction between learner-instructor, learner-learner, learner-content and learner-outside resources; integration of formative and summative assessment mechanisms for learners and instructors.

\section{Social networking}

Social networking is a tool being explored by many institutions as a means of connecting to and communicating with students. It is made possible in the modern era through communication networks, where using online contacts people communicate each other and access information that they require. Educators all over the world hold virtual office hours on Facebook and Twitter and, they post assignments or run discussions using WhatsApp Viber and Skype.

\section{Assumptions}

In DA the examiner interferes with the issue the learner is about to solve so as to make out the strategies and processes of mental functioning (Macrine and Lidz, 2001). Accordingly, there is no attempt to prejudge the cooperation as to what previous experience the learner has experienced. Learners are observed not merely in their actuality, but in their potentiality as well. One of the DA's fundemantal objectives is is to shift the person and realize the potential for change in the course of interaction.

Gillam and Pena (2004) state that the use of DA increases the clarification of ability among diverse ethnic backgrounds so the idea of a democratic educational system could benefit immensely from DA.

Haywood and Lidz (2007) argue that DA's interactive mood objectively measures the probability of change by means of different methods. However, while designing a curriculum, the factors such as time, cost, and knowledge of the teachers may curb the practice of the DA approach commonly.

\section{The Problem of The Study}

To build a structured mechanism for the validity of DA, Poehner introduces the micro/macro framework of validity for DA. As to Poehner (2011b), "while micro validity points to specific events of mediator-learner cooperation during a DA session, macro validity accepts patterns throughout the session" (p. 259).

In order to examine the ability we tried to find out the answers to these questions:

(a) How should one specify the size of overlapping ZPDs of different grammatical points?

(b) If it is maintained that the group DA has also the required validity, how can the framework indicate the development in a large class size?

\section{Conclusion}

The integration of EFL, CALL and E-B-M learning can help teachers and learners to accommodate a wide variety of learning needs of all types of learners, visual, oral, kinesthetic etc. In this study we attempted to explain and analyze DA in the use of foreign language 
teaching but there stil exist plenty of questions wating to be answered by the researchers. The self-assessment of knowledge within our field of research can help us in the improvement of the field. The study also shows that DA approaches of development and assessment are fruitful and productive in terms of psychoeducational assessment. It is obvious that the interdisciplinary research can contribute to the better understanding of the instances.

The results of this study indicate that dynamic virtual environments are quite significant in foreign language education. ICT has become part and parcel of teachers' academic life that they employ in teaching, research, academic and administrative communication. The familiarity and competence of the students in CALL allow them to a possess a cutting edge in an ICT based learning environment. EFL teachers need to consider these factors in defining their classroom role and, encourage the integration of CALL by providing students a collaborative and autonomous learning that empowers them to access, retrieve and store, and exchange information. These new modes of teaching and learning should be integrated into our higher education sector to be at pace with the fast changing technology.

\section{References}

Felix, U. (Ed.). (2003). Language learning online: Towards best practice.

Gillam, R. B, \& Pena, E. D. (2004). Dynamic assessment of children from culturally diverse backgrounds. Communication Disorders and Science in Culturally and Linguistically Diverse Populations, 11(1), 2-5.

Haywood, H. C. (1992). Interactive assessment: A special issue. Journal of Special Education, 26(3), 233-234.

Luria, A. R. (1961). Study of the abnormal child. American Journal of Orthopsychiatry. Journal of Human Behavior, 31, 1-13.

Lantolf, J. P., \& Poehner, M. E. (2004). Dynamic assessment: Bringing the past into the future. Journal of Applied Linguistics, 1(1), 52-70.

Macrine, S. L., \& Lidz, C. S. (2001). An alternative approach to the identification of gifted culturally and linguistically diverse learners: The contribution of dynamic assessment. School Psychology International, 22(1), 74-96.

Mehri, E. \& Amerian, M. (2015). Challenges to Dynamic Assessment in Second Language Learning. Theory and Practice in Linguistic Studies. 5(7), 1461-1463.

Poehner, M. E. (2011b). Validity and interaction in the ZPD: Interpreting learner development through L2 dynamic assessment.

Valinser, J., \& van der Veer, R. (eds.) (1993). The encoding of distance: The concept of the zone of proximal development and its interpretation. In R. Cocking and A. Renninger. The development of meaning and psychological distance. Hillsdale, N. J.: Lawrence Erlbaum.

Vyogtsky, L. (1986). Language and Thought. USA: MIT.

Zhang, Y. (2013). The theoretical construction of dynamic assessment mode in Chinese tertiary EFL writing class with online teaching and scoring system. CALL-EJ, 14(2), $38-50$ 